\title{
Government and industry investment plays crucial role in further establishment, evolution, and diversification of insect agriculture: a case example from the United States
}

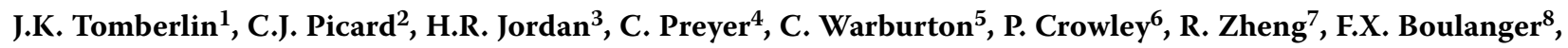 \\ I. Banks 9 , M. Lefranc ${ }^{10}$, M.J. Zorrilla ${ }^{11}$, G. Olson ${ }^{12}$, G. Aid ${ }^{13}$, D. Fluker ${ }^{14}$, B.J. Bench ${ }^{15}$, M. Teo ${ }^{16}$, C.S. Richards ${ }^{17}$, \\ C. Jones ${ }^{18}$, J. Gonzalez ${ }^{19}$ and M. Eljendy ${ }^{20}$
}

${ }^{1}$ Department of Entomology, Texas AEM University, Texas, USA; ${ }^{2}$ Department of Biology, Indiana University-Purdue University in Indianapolis, Indiana, USA, ${ }^{3}$ Department of Biology, Mississippi State University, Mississippi USA; ${ }^{4}$ National Science Foundation Center for Environmental Sustainability through Insect Farming, Texas, USA; ${ }^{5}$ Entosystem, Quebec, Canada; ${ }^{6}$ Chapul Farms, Oregon, USA; ${ }^{7}$ Protix, the Netherlands; ${ }^{8}$ Agronutris, France; ${ }^{9}$ PreZero US, Inc., California, USA; ${ }^{10}$ InnovaFeed, France; ${ }^{11}$ Nutrition Technologies, Malaysia; ${ }^{12}$ Enterra, Canada; ${ }^{13}$ Ragn-Sells, Sweden; ${ }^{14}$ Soldier Fly Technologies, Louisiana, USA; ${ }^{15}$ Tyson Foods, Inc., Ingredient Solutions Group, Arkansas, USA; ${ }^{16}$ Entofood, Malaysia; ${ }^{17}$ Veolia North America, Massachusetts, USA; ${ }^{18}$ Mars Inc., California, USA; ${ }^{19}$ Phileo by Lesaffre, France; ${ }^{20}$ Proteinea, Egypt; jeffery.tomberlin@ag.tamu.edu

(c) 2022 Wageningen Academic Publishers

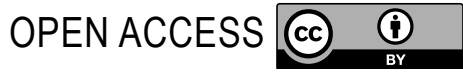

EDITORIAL

Insect farming for both food and feed has become an established part of agriculture globally in response to demand for protein and commitment to more sustainable agricultural practices. Large international companies are diversifying to capture this opportunity, and start-up companies are rapidly developing to meet the increasing demand. In response, research within universities and companies is promptly expanding, resulting in challenges faced by all - industry, academia, and regulatory bodies. How does one keep up with the massive amount of information being generated annually? How can such entities collaborate to prevent redundancy and increase efficiency while identifying knowledge gaps and avoiding pitfalls known and unknown?

Governments, as well as industry, are investing considerable resources in the development of the insect-farming sector globally. For example, the European Union has made substantial investments while simultaneously developing regulations to assimilate the industry safely and effectively into regional economies. Such efforts have led to clear guidelines for companies desiring to engage economic opportunities in a particular region. Of course, the economic benefits are evident; however, the associated benefits beyond financial gain are also significant drivers, such as developing circular economies, protecting the environment, and bolstering sustainability. Limitations constraining government influence are also evident. Financial investment is a bottleneck restricting the industry's rate to diversify, grow, and stabilise. Government investments in research or corporate development typically operate within a bubble with limited cross-collaboration. Similar challenges exist for governments globally. We present an example of government investment in the United States that could serve as a global synergetic opportunity for the insects as a food and feed industry.
The National Science Foundation (NSF), a U.S. federal agency with a global reputation for supporting groundbreaking research in science, engineering, and learning has a flagship program that could address, in part, such challenges being experienced globally. Through the Industry-University Cooperative Research Centers (I/ UCRC) Program, the NSF is investing \$2.25M USD in creating the Center for Environmental Sustainability through Insect Farming (CEIF) ${ }^{1}$.

An IUCRC operates as a consortium integrating industry, government, and academia. The industrial community includes major corporations, middle market companies, small businesses, and start-ups; public participants may

\footnotetext{
${ }^{1}$ https://www.nsf.gov/awardsearch/showAward?AWD_ID =2052788 \&HistoricalAwards=false;

https://www.nsf.gov/awardsearch/showAward?AWD_ID=2052454\& HistoricalAwards=false;

https://www.nsf.gov/awardsearch/showAward?AWD_ID=2052565\& HistoricalAwards=false.
} 
range from local governments to divisions of federal agencies. These stakeholders partner to advance critical technologies from early stage research to the marketplace. The membership fees support research projects conducted by university faculty and students. NSF provides funding for operations and a governance framework for membership, operations, and evaluation.

IUCRCs engage in basic research that is considered 'precompetitive.' This means that the research is intended for publication through avenues available for academic and scientific research. Pre-competitive research allows industry Members who might be competitors in the commercial marketplace to work collaboratively within an IUCRC to help shape its research activities and focus. IUCRCs are expected to work on research that is transformative and helps reshape the frontiers of knowledge of industry relevance.

An IUCRC provides the infrastructure for training a diverse, high-tech workforce, including through the recruitment of students and faculty of populations historically underrepresented in science, technology, engineering, and mathematics (STEM) disciplines. Furthermore, it provides a forum for start-ups to engage with industry and develop a rich network of corporate, academic, and public resources to accelerate technology translation.

The CEIF is a collective effort across Texas A\&M University AgriLife (TAMU), Indiana University-Purdue University in Indianapolis (IUPUI), and Mississippi State University (MSU). Each site has a crucial platform for research, with TAMU focusing on optimising insect production and conducting feed trials for aquaculture, poultry, and pets. IUPUI is concentrating on the genetics/genomics of massproduced insects for the food and feed sector. And MSU is primarily engaged with research relevant to microbiology, quality assurance, and optimisation of production. These pillars of research are only the beginning as the CEIF will continually evolve to meet the needs of its industry partners. This evolution includes diversifying research conducted through site additions and including other insect models. Furthermore, the CEIF has the opportunity to collaborate with other academic institutions located throughout the world depending on the needs of the industry.

The NSF IUCRC program presents numerous benefits to participating industry partners. Industry partners are part of the Industry Advisory Board, which reviews and recommends projects for funding from an industry perspective. Academia also benefits through training opportunities for students in a new potential employment sector, novel research, and the scientific discovery process that underpins academic researchers' passions. With a small financial investment, industry members have access to a tremendous amount of data generated annually as they get access to the full data generated across all projects. For example, a company contributing $\$ 25,000$ in membership dues annually over five years would have access to a minimum of $\$ 4.5$ million investment in research. Furthermore, based on NSF guidelines, industry partners can license intellectual property with no royalties returned to the universities. Moreover, funds invested by industry are used primarily for research as only $10 \%$ is returned to the universities to cover overhead.

Industry partners can collaborate through the Industry Advisory Board, where they are presented with the opportunity to communicate with one another through two annual meetings and through other channels outside of the meetings. A grassroots approach to incorporate engagement naturally leads to synergy, collaboration, and momentum for informed growth and expansion. Given that funds for research can often be a limiting factor for many companies in the insects as food and feed sector, the CEIF presents a low risk and high reward opportunity for companies involved.

The NSF IUCRC program creates research value within universities to industry. Of course, the initial NSF commitment of $\$ 2.25$ million is substantial and will provide administrative support to the CEIF. However, universities also gain insights into the industry's critical challenges and could lead to new grant opportunities with industry partners or other researchers. At a minimum, small investments annually result in rapid data generation addressing current matters faced by the industry. Secondarily, the industry is presented with the opportunity to engage students and postdoctoral associates conducting the research, leading to the next generation of staff working within their facilities conducting confidential research, optimising processes, and enhancing quality assurance.

The NSF IUCRC program also creates value for governments. This impact is most evident when one considers the needs of the industry to engage government agencies about regulatory approval of insect-based products. Industry can develop a 'roadmap' for collecting data necessary to address concerns various government agencies have with products for human or animal consumption, soil amendments, and sustainability. Furthermore, centres, such as the CEIF, can engage commodity groups already communicating with regulatory bodies to formulate a concise and targeted collaboration resulting in efficient review and potential approval of a commodity.

The mission of the CEIF is to develop a global platform for the insects as food and industry to expand, diversify, and stabilise the insect agriculture sector. Following the model developed by the NSF, the CEIF will be able to bridge with universities throughout the world. As the Industry Advisory Board identifies topics of research importance, the CEIF 
leadership will seek out researchers at their respective universities and those located near the industry partners that can address such matters. This approach allows for the CEIF to have maximum flexibility to address the needs of the industry. Furthermore, given the quick turnaround (i.e. two meetings annually with the summer meeting used to identify research needs and present results from the previous year, while the second is presenting new research projects to be considered for funding), data are generated and provided to the industry advisory board members in a manner that allows for industrial growth opportunities as needed to maximise their financial investment in the sector.

The CEIF is open to researching not only the 'big three' (e.g. black soldier fly, mealworm, cricket), but also any other insect models or topics deemed necessary by the Industry Advisory Board. The industry has gravitated towards these three primary insects for mass production but continues to diversify in terms of insect models as well as outputs beyond protein, such as insect frass and bioenergy. However, the primary mission of the CEIF is to conduct research that addresses areas of concern identified by its industry members (e.g. pathogen identification and prevention, optimisation of output, breeding selective lines for targeted production value). As the industry continues to evolve, research will do so as well with potential endeavours into economics, environmental sustainability, chemistry, and much more. The sustained CEIF engagement between industry partners and academia will generate breakthrough research that will expand the sector and provide important basic science of industry relevance.

\section{Acknowledgements}

We would like to thank A. van Huis and P. Balan for feedback on earlier versions of this manuscript. 
